\title{
De l'eau en abondance
}

Françoise Verrey Bass

Correspondance:

Dr Françoise Verrey Bass Plänkestrasse 12

CH-2502 Bienne

fraverrey[at]gmx.ch
Nous nous trouvons au pied d'un monticule surmonté d'un immense socle sur lequel galopent 10 chevaux en bronze grandeur nature. Pour être exact, nous ne sommes pas encore au pied du monticule, mais à l'entrée d'un parc géant, très vert, très fleuri, avec des allées toutes pavées, larges et des ruisseaux qui longent ces allées, une eau claire, transparente, fraîche, au débit rapide. Nous voyons le monticule à environ 200 mètres et plus près, la statue d'un homme haute d'environ dix mètres, en or.
d'Ashgabat ne nous tente guère. Nous sommes dans la capitale du Turkménistan, construite à la gloire de Turkmanbashy, le président dictateur depuis que les Russes ont reconnu l'indépendance du pays au début des années 90 et sont partis.

Nous sommes depuis hier dans ce pays qui se compose en gros de deux oasis, l'une avec la ville de Mary ou Merw et l'autre justement la capitale. Le reste n'est quasi que désert, le très fameux Karakoum de la Route de la Soie.

\section{«ll faut simplement, comme toujours, être dans la bonne barque, celle du président.»}

Il y a 5 minutes nous avons traversé une place avec en son centre un socle sur lequel est posé un livre géant ouvert avec des pages en or. Pendant notre tournée en bus, une ambulance nous suit. Elle nous suivra partout, toute la journée. Dedans deux hommes en blouse blanche, l'un avec le stéthoscope autour du cou. "Au cas où quelqu'un se sentirait mal», nous a dit la guide en réponse à notre question. Mais bien sûr, c'est la police secrète, nous serons toute la journée sous surveillance rapprochée. L'un d'entre nous pose une question un peu osée. La guide nous prie instamment de ne pas faire de commentaires, pas de remarque, de nous tenir tranquilles. Et nous qui voulions jouer la comédie d'un évanouissement sur la place. Pas malin! C'est elle qui aura des ennuis! Puis atterrir dans l'unique hôpital

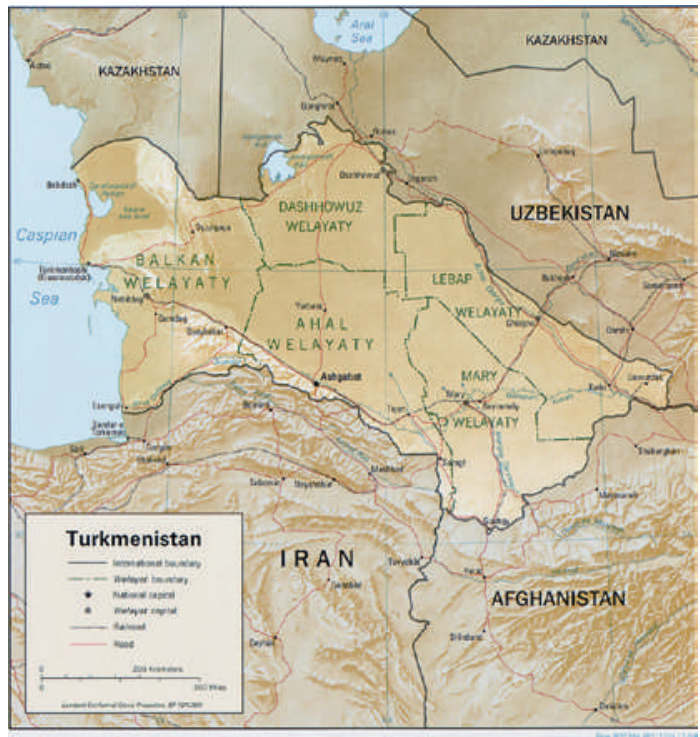

Nous venions de l'Ouzbékistan, un vrai paradis de liberté comparé à ce que l'on ressent ici et même l'Iran qui sera notre prochain pays n'est de loin pas aussi muselé. A la frontière du Turkménistan nous avons quitté notre bus ouzbèk à $1 \mathrm{~km}$ au moins de celle-ci. Chacun a du prendre ses bagages en charge et, un à un, nous avons suivi un couloir ouvert vers le ciel, mais avec murs et barbelés des deux côtés, sous un soleil de midi impitoyable, tombant à la verticale, jusqu'à la maisonnette de la frontière où se trouvaient déjà quelques indigènes. Alors l'attente commença. Nous avions donné passeports et visas. Nous attendions. Pas une feuille pour nous abriter du soleil implacable, pas de sièges, une seule barrière en bois comme possibilité de s'appuyer à tour de rôle un moment. Le sol était tellement poussiéreux, inutile de songer même à se poser. Pas de toilettes. A la limite, c'était égal, nous n'avions rien à boire et nous transpirions abondamment.

Enfin, à la queue leu leu, nous avons pu passer devant le guichet des gardes frontières au visage fermé et nous avons été pris en charge par notre nouveau guide, une femme grande et forte, mais sensible à pleurer pour un rien, une turkmène russe.

Laissant la frontière derrière nous, prochaine surprise: la guide nous fait descendre du bus pour traverser à pied un pont fait de pontons datant de l'époque russe, devenu trop faible pour transporter bus et passagers. En marchant il faut éviter les trous et fentes assez larges dans la vieille ferraille rouillée de la passerelle posée sur les pontons. Dessous coule lentement une eau sale, brune, avec ici et là de la mousse jaunâtre. Ça, l'immense fleuve Amudar'ja?

Et maintenant ce superbe parc avec des jets d'eau, des ruisseaux partout. L'eau canalisée ou libre qui descend de tous les côtés depuis le sommet du 
monticule, glougloutante ou cascadante et qui longent les pelouses vertes arrosées en continu par des jets d'eau supplémentaires. D'autres statues géantes. Tout autour du parc nous voyons un quartier de grandes maisons d'habitation genre tours avec des appartements bien visibles au travers d'énormes baies vitrées. Des appartements neufs, mais il n'y a aucun signe d'aménagement en cours des pelouses vertes tout autour. Il s'agit vraisemblablement de maisons âgées de deux à trois ans, presque toutes complètement vides, un quartier fantôme. Un quartier pour les riches, car il y en a. Le Turkménistan est très riche en pétrole et en gaz. Il faut simplement, comme toujours, être dans la bonne barque, celle du président.

En 1954 Les Russes ont commencé la construction du canal Karakoum en déviant les eaux du fleuve Amudar'ja qui partait vers l'Ouzbékistan pour aller se jeter - et alimenter - la mer Aral. Depuis la fin de la construction du canal, qui rejoint la mer Caspienne, et surtout depuis la construction dès l'an 2000 d'un lac artificiel dans la plaine du Karakoum, le fleuve devint de plus en plus étroit. Il disparaît lors de sécheresse complètement dans le sable. Les poissons ont disparus. Les rives sont ensablées. Tandis que le long du canal les champs de cotons et de riz prospèrent et qu'Ashgabat est devenue la ville des superlatifs avec même un port, donc une navigation de plaisance et de transports jusqu'à la mer Caspienne.

D'accord, Saparmyrat Nyýazow, alias Turkmanbashy, président à vie jusqu'en 2006, n'a fait que continuer l'entreprise des Russes et que construire des monuments à sa gloire éternelle...

N'ayant pas de descendants, c'est son médecin personnel qui est devenu président à sa mort, élu unanimement par le conseil des ministres. Ah, un

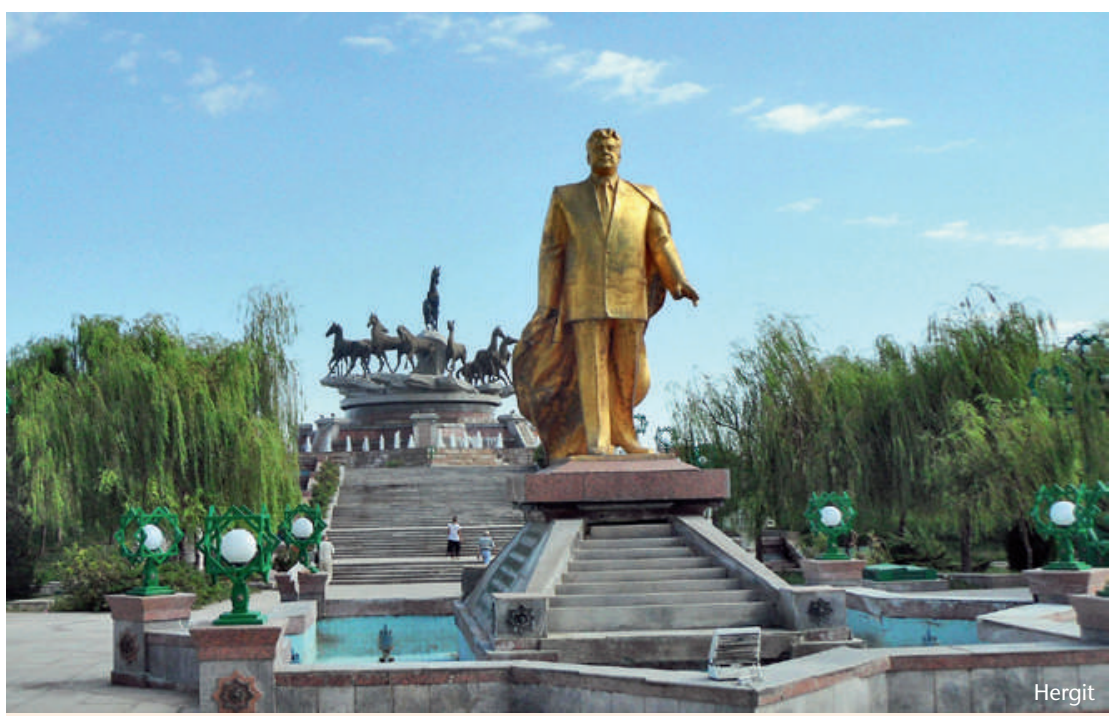

Turkmanbashy n'a fait que continuer l'entreprise des Russes et que construire des monuments à sa gloire éternelle. détail, en 2003 Turkmanbashy se fit déclarer «le Prophète» par son cabinet.

Turkmanbashy président a interdit tout théâtres et opéras, ainsi que le libre choix des études. Il a écrit des livres, dont l'un, la Ruhnama, est lecture scolaire obligatoire pour tous, le fameux livre en or sur l'une des places de la ville, proche du Palais présidentiel, que l'on ne peut pas voir depuis la rue et duquel on ne peut pas s'approcher. La Ruhnama prescrit ce qui est autorisé ou ne l'est pas. Avantage: il est depuis toujours défendu de fumer en public et de jeter la moindre des choses par terre ou de cracher. Interdit d'avoir un animal domestique chez soi en ville. L'habillement doit toujours être correct.

Ainsi, la ville est très propre et l'on ne voit nulle part d'attitude douteuse, négligée, agressive. La criminalité? Elle n'existe pas. Mais les droits de l'homme et la liberté d'expression non plus. La religion est musulmane et russe orthodoxe. Des minorités chrétiennes sont autorisées, mais pas les juifs. La peine de mort n'existe plus depuis 2000, mais les opposants au régime, s'il y en a, atterrissent vite en prison à vie ou fuient leur pays.

\section{«C'est la nature qui décide de la longévité des gens.»}

Turkmanbashy a réduit toutes les dépenses sociales et fermé tous les hôpitaux et dispensaires du pays, à part l'hôpital d'Ashgabat, qui se spécialise aujourd'hui paraît-il dans les problèmes de fertilisation in vitro pour une riche clientèle étrangère qui de cette manière contourne les lois de leur propre pays. Par contre, il est intéressant de savoir que certains produits sont gratuits pour tous: sel, eau, électricité et gaz. Le pain et la benzine sont très bon marché. Mais sans médecine, pas de problèmes de budget social, de santé et de gériatrie. C'est la nature qui décide de la longévité des gens.

Combien de temps le régime arrivera-t-il encore à exclure le pays de toute influence extérieure? Surtout que le médecin-président avait promis certaines réformes comme les votations libres (un seul parti, pas d'opposition, entre 85 et $99 \%$ de participation aux votes!) et l'instauration de l'Internet. On ne sait pas, si cela s'est fait.

En quittant Ashgabat pour l'Iran, nous passons le seul point de frontière existant entre ces deux pays. Les montagnes sont garnies de rangées de fil de fer barbelé! Il y a partout des miradors. Des soldats patrouillent des deux côtés de la frontière. Mais après un nouveau passage de frontière assez difficile, nous sommes reçus par des hommes et des femmes souriants et aimables. Nous sommes vraiment contents de quitter l'atmosphère lourde du Turkménistan. 